\title{
Adipose tissue-skeletal muscle crosstalk: are endocannabinoids an unwanted caller?
}

\author{
M. J. Watt
}

Received: 23 December 2008 / Accepted: 20 January 2009/Published online: 10 February 2009

(C) Springer-Verlag 2009

Keywords Adipose tissue · Endocannabinoids . Insulin resistance $\cdot$ Skeletal muscle

\author{
Abbreviations \\ AEA Anandamide \\ CB1R Cannabinoid type 1 receptor \\ ECS Endocannabinoid system \\ JNK c-Jun N-terminal kinase
}

Adipose tissue was historically regarded as an inert depot for triacylglycerol storage; however, it is now recognised as a major endocrine organ that is central to whole-body metabolic homeostasis. The adipocyte exerts its effects on lipid and carbohydrate use by producing and releasing peptides/hormones that signal to other tissues to convey biological signals. Indeed, the concept of metabolic 'crosstalk' between adipose tissue and other key organs that regulate metabolism is the subject of much research.

Obesity is characterised by adipocyte hypertrophy, an expanded visceral and subcutaneous adipose tissue mass and altered cellular biology [1]. While obesity is undoubtedly a major risk factor for insulin resistance, the factors linking these pathologies are not well defined. Currently, there are two prevailing views to explain this link. The lipocentric view is that excessive lipolysis in an expanded adipose tissue mass results in an oversupply of NEFA to peripheral metabolic tissues that are not equipped to store excess lipids. The resulting ectopic lipid deposition activates serine/

M. J. Watt $(\bowtie)$

Department of Physiology, Monash University,

Building 13F,

Clayton, VIC 3800, Australia

e-mail: matthew.watt@med.monash.edu.au threonine kinases (i.e. c-Jun N-terminal kinase [JNK], inhibitor of $\mathrm{kB}$ kinase, conventional protein kinase $\mathrm{C}$ ) that directly disrupt insulin signal transduction [2]. In severe cases, ectopic lipid accumulation induces lipotoxic cell death [3]. An alternative view is that obesity generates a chronic low-grade inflammatory response. This is characterised by abnormal cytokine production (e.g. TNF $\alpha$, IL-6), mostly in adipocytes, but also in macrophages that are recruited to, and reside in, adipose tissue. These, in turn, activate the aforementioned serine/threonine kinases in metabolic tissues to inhibit insulin action [4]. The likelihood is that both mediators, and others, contribute to insulin resistance in human obesity.

In this issue of Diabetologia, Eckardt and colleagues [5] have provided new evidence that the endocannabinoid system (ECS) is directly involved in the negative crosstalk between adipose tissue and skeletal muscle, thereby adding endocannabinoids to the growing list of adipocyte-derived factors that mediate skeletal muscle insulin resistance. Endocannabinoids are a family of lipid ligands derived from cell membrane phospholipids that bind and activate the type 1 and type 2 cannabinoid receptors (CB1R and CB2R), encoded by $C N R 1$ and $C N R 2$, respectively. Stimulation of the endocannabinoid system, mainly via CB1R, increases food intake, promotes weight gain, induces lipogenesis in liver and adipose tissue and causes whole body glucose intolerance [6]. The ECS is overactivated in obesity [7], and studies in $\mathrm{Cnrl}^{-/-}$mice reveal a lean phenotype that is resistant to diet-induced obesity [8]. Although endocannabinoids are implicated in the development of obesity through central and peripheral mechanisms, the tissue-specific contribution of the ECS to metabolic regulation, particularly in skeletal muscle, is incompletely described. 
Using skeletal muscle myotubes originating from human biopsies, Eckardt et al. [5] reported that the endogenous endocannabinoid, anandamide (AEA), decreases insulinstimulated glucose uptake at high doses and that the addition of a specific CB1R antagonist to muscle cells restored insulin sensitivity. Unsurprisingly, the insulin resistance generated by AEA in muscle cells was associated with an attenuation of insulin signal transduction. Interestingly, though, there was selectivity in this suppression, with evidence of enhanced IRS1 (Ser 307) phosphorylation and reduced Akt (Ser 473) phosphorylation (both inhibitory), yet no effects on Akt (Thr 308) or glycogen synthase kinase $3 \alpha / \beta$ phosphorylation were observed. The authors also assessed some potential mediators of the insulin resistance. AEA rapidly increased phosphorylation of extracellular regulated kinase $1 / 2$ and p38 mitogen-activated protein kinase; however, the contribution of these kinases to insulin resistance was not established (Fig. 1). JNK activation, which has consistently been implicated in the development of skeletal muscle insulin resistance, was not affected. These findings are in contrast to those of previous work showing that endocannabinoids activate the phosphatidylinositol 3-kinase/Akt signalling pathway in other cell types and enhance the sensitivity of adipocytes to insulin [9]. In light of this, the relatively long treatment with AEA could result in transcriptional activation of lipid synthesis pathways and intracellular lipid accumulation, as occurs in adipocytes [10]. The difference here is that adipocytes have evolved to efficiently store lipids, whereas the deposition of lipid metabolites such as ceramide and diacylglycerol cause insulin resistance in muscle.

Next, the authors established a link between adipose tissue endocannabinoid secretion and skeletal muscle insulin resistance. To achieve this, primary adipocytes were generated from human adipose tissue, and muscle cells were treated with conditioned media from these adipocytes. Not surprisingly, the conditioned media induced insulin resistance in muscle but, importantly, treating cells with the selective CB1R antagonist rimonabant partially reversed the insulin resistance. Perhaps the most significant aspect of these experiments was that the conditioned media contained endocannabinoids at concentrations $\sim 300-1,000$ times below those detected in human plasma $(\sim 3 \mathrm{nmol} / \mathrm{l})$ and in the aforementioned AEA experiments. This implies that CB1R is extremely sensitive to a complex mixture of endocannabinoids, rather than one endocannabinoid in isolation, and/or that interactions of endocannabinoids with other adipocytereleased factors enhance CB1R signalling. Another unexpected finding was that AEA enhanced basal glucose uptake and that rimonabant decreased glucose uptake in muscle cells. These data indicate that insulin-independent signalling for glucose uptake in muscle is enhanced by the ECS, but is unlikely to involve the well-studied putative glucose-lowering target protein 5'-AMP-activated protein kinase [11, 12].

Several observations in this paper have added new knowledge to our understanding, not only of the factors regulating skeletal muscle insulin resistance, but also of the involvement of adipose tissue as a pathogenic organ. The

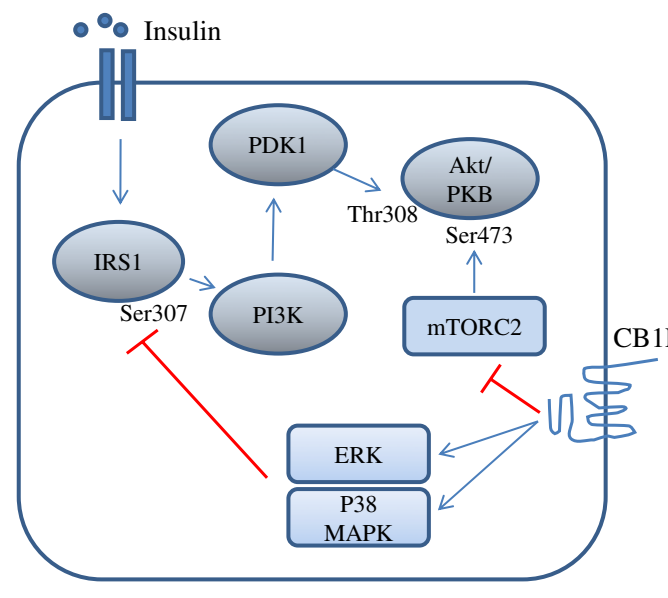

Skeletal muscle

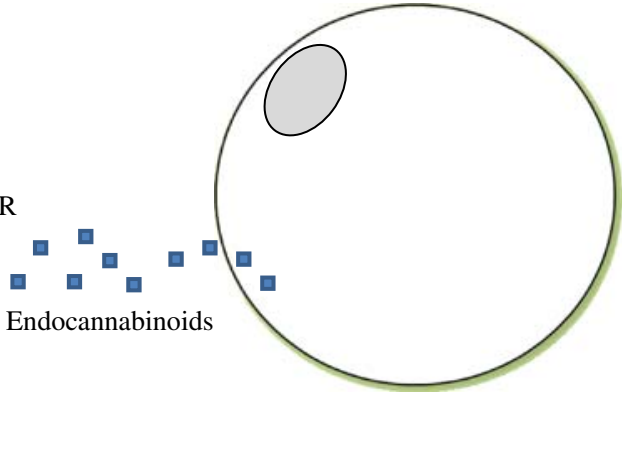

Adipocyte
Fig. 1 Simplified model outlining the putative cellular events mediating the downregulation of insulin action by endocannabinoids. Insulin binding to the insulin receptor triggers a signalling cascade involving tyrosine phosphorylation of IRS1, activation of phosphatidylinositol 3-kinase (PI3K), resulting in an increase in phosphatidylinositol-3,4,5-trisphosphate, which is responsible for the recruitment of Akt to the plasma membrane alongside PDK1 phosphatidylinositol-dependent kinase 1 (PDK1). It is here that Akt is phosphorylated at Thr308 (activation loop site) by PDK1 and at
Ser473 (hydrophobic motif site) by mammalian target of rapamycin complex 2 (mTORC2). Release of endocannabinoids from adipocytes and activation of CB1R in muscle activates extracellular regulated kinase (ERK) and p38 mitogen-activated protein kinase (MAPK), which, in turn, leads to phosphorylation of IRS1 Ser307 and inhibition of insulin signalling. CB1R activation is also hypothesised to blunt mTORC2 activation, thereby attenuating phosphorylation of Akt Ser473 
findings of Eckardt et al. [5] corroborate previous work in mice, which identified the ECS system as an important component of adipose tissue [13], and supports the view that dysregulation of the ECS in obesity may contribute to skeletal muscle insulin resistance. It also highlights a previously unappreciated direct role of endocannabinoids in skeletal muscle glucose metabolism, independent of metabolic alterations associated with weight loss or changes in other hormones known to influence nutrient metabolism (e.g. adiponectin). From a clinical viewpoint, the data indicate that peripheral therapeutic targeting of the ECS may prove effective at reducing the development of insulin resistance and type 2 diabetes, independent of central nervous system effects, such as anxiety, depression and nausea, that have been linked to CB1R antagonist/inverse agonist treatment $[14,15]$. In this regard, the first approved selective CB1R agonist, rimonabant, was recently suspended by the European Medicines Agency due to the risk of serious psychiatric problems, suggesting specific problems with the target/pathway (i.e. CB1R) or off-target effects of the drug. Caution will be paramount to any future therapeutic strategy involving CB1R.

Despite this initial step towards understanding the role of the adipocyte-derived endocannabinoids in regulating skeletal muscle glucose homeostasis, much remains to be learned. The factors driving endocannabinoid production in, and release from, adipose tissue in obesity/type 2 diabetes are unresolved, and the contribution of adiposederived endocannabinoids to plasma levels in vivo needs to be established. Individuals with diabetes store more intermuscular fat [16], and given the remarkable sensitivity of the CB1R to the adipocyte endocannabinoid mixture described by Eckardt et al. [5], local paracrine signalling could mediate muscle insulin resistance in vivo. A detailed understanding of the cellular and molecular mechanisms by which ECS activation causes skeletal muscle insulin resistance awaits investigation, as does the potential role of the other cannabinoid receptor, CB2R. Knowledge of these pathways may lead to specific therapeutic manoeuvres that bypass CB1R, which may, in turn, circumvent the neural affects that have led to the demise of central nervous system CB1R antagonists as viable therapeutics. Based on our current knowledge of CB1R signalling, one would predict the involvement of serine/threonine kinases and, possibly, excessive lipid metabolite deposition. Some appreciation of prolonged ECS activation and, most importantly, in vivo validation of muscle-specific effects, are important future directions.

Given the beneficial metabolic effects of CB1R antagonists, independent of weight loss, further dissection of the roles of the ECS in skeletal muscle and other metabolic tissues will provide important insights into the therapeutic promise this pathway holds for insulin resistance and type 2 diabetes.
Acknowledgements M. J. Watt thanks M. Febbraio (Baker IDI Heart and Diabetes Institute, Prahran, VIC, Australia) and B. Oldfield (Monash University, Clayton, VIC, Australia) for their thoughts and critical reading. M. J. Watt is supported by an R. Douglas Wright fellowship from the National Health and Medical Research Council of Australia.

Duality of interest The author declares that there is no duality of interest associated with this manuscript.

\section{References}

1. Bays HE, Gonzalez-Campoy JM, Bray GA et al (2008) Pathogenic potential of adipose tissue and metabolic consequences of adipocyte hypertrophy and increased visceral adiposity. Expert Rev Cardiovasc Ther 6:343-368

2. Savage DB, Petersen KF, Shulman GI (2007) Disordered lipid metabolism and the pathogenesis of insulin resistance. Physiol Rev 87:507-520

3. Unger RH (2003) Minireview: weapons of lean body mass destruction: the role of ectopic lipids in the metabolic syndrome. Endocrinology 144:5159-5165

4. Weisberg SP, McCann D, Desai M, Rosenbaum M, Leibel RL, Ferrante AW Jr (2003) Obesity is associated with macrophage accumulation in adipose tissue. J Clin Invest 112:1796-1808

5. Eckardt K, Sell H, Taube A et al (2009) Cannabinoid type 1 receptors in human skeletal muscle cells participate in the negative crosstalk between fat and muscle. Diabetologia. doi:10.1007/ s00125-008-1240-4

6. Cota D (2007) CB1 receptors: emerging evidence for central and peripheral mechanisms that regulate energy balance, metabolism, and cardiovascular health. Diabetes Metab Res Rev 23:507-517

7. Bluher M, Engeli S, Kloting N et al (2006) Dysregulation of the peripheral and adipose tissue endocannabinoid system in human abdominal obesity. Diabetes 55:3053-3060

8. Cota D, Marsicano G, Tschop M et al (2003) The endogenous cannabinoid system affects energy balance via central orexigenic drive and peripheral lipogenesis. J Clin Invest 112:423-431

9. Motaghedi R, McGraw TE (2008) The CB1 endocannabinoid system modulates adipocyte insulin sensitivity. Obesity (Silver Spring) 16:1727-1734

10. Bellocchio L, Cervino C, Vicennati V, Pasquali R, Pagotto U (2008) Cannabinoid type 1 receptor: another arrow in the adipocytes' bow. J Neuroendocrinol 20(Suppl 1):130-138

11. Kola B, Hubina E, Tucci SA et al (2005) Cannabinoids and ghrelin have both central and peripheral metabolic and cardiac effects via AMP-activated protein kinase. J Biol Chem 280:25196-25201

12. Watanabe T, Kubota N, Ohsugi M et al (2009) Rimonabant ameliorates insulin resistance via both adiponectin-dependent and adiponectin-independent pathways. J Biol Chem 284:1803-1812

13. Di Marzo V (2008) The endocannabinoid system in obesity and type 2 diabetes. Diabetologia 51:1356-1367

14. Christensen R, Kristensen PK, Bartels EM, Bliddal H, Astrup A (2007) Efficacy and safety of the weight-loss drug rimonabant: a meta-analysis of randomised trials. Lancet 370:1706-1713

15. Van Gaal L, Pi-Sunyer X, Despres JP, McCarthy C, Scheen A (2008) Efficacy and safety of rimonabant for improvement of multiple cardiometabolic risk factors in overweight/obese patients: pooled 1-year data from the Rimonabant in Obesity (RIO) program. Diabetes Care 31(Suppl 2):S229-S240

16. Miljkovic-Gacic I, Gordon CL, Goodpaster BH et al (2008) Adipose tissue infiltration in skeletal muscle: age patterns and association with diabetes among men of African ancestry. Am J Clin Nutr 87:1590-1595 